\title{
Cross-talk effects on the Regenerator Allocation optimization*
}

\author{
Eva Marín-Tordera, Xavier Masip-Bruin, Marcelo Yannuzzi, \\ René Serral-Gracià, Sergio Sánchez López
}

\begin{abstract}
Advanced Network Architectures Lab (CRAAX), Universitat Politècnica de Catalunya (UPC)
$\{$ eva, xmasip, yannuzzi, rserral, sergio\}@ac.upc.edu
\end{abstract}

Abstract - This paper studies the impact of considering crosstalk effects in the IA-RWA process and in the regenerator allocation algorithms. First of all, we evaluate if the regenerator allocation optimization is still useful when the crosstalk effects are considered. For this end we improve a simple physical model which computes the quality of the signal factor for each optical connection. Until now this physical model was unaware of the physical layer impairments depending on the interference between different optical connections, such as the crosstalk. Following the same simple methodology we add the switching node crosstalk effect to the computation of the quality of the signal factor.

Once the new physical model is enhanced we evaluate the impact of the crosstalk on the performance of IA-RWA algorithms that optimize the use of the regenerators; and also we evaluate the impact of different e wavelength assignation techniques.

\section{INTRODUCTION}

Optical Transport Networks (OTNs) based on WavelengthDivision Multiplexing (WDM) have achieved today a high degree of maturity. With the extremely rapid growth of Internet traffic generated by new applications and subscriber needs, a new challenge awaits OTN: that is the capability of scalingup in capacity, while keeping the cost per transported bit at the same level or even lowering it.

On one hand, the rapid evolution of photonic technology has brought to the market devices capable of performing transparent optical switching. Transparent optical networks do not convert the signal to the electrical domain in the intermediate nodes, but rather keep the signal within the optical domain from the source node to the destination node. However, full transparency is not always achievable in long distance networks due to the degradation of the optical signal. It is well recognized that one of the major limitations in this process is represented by the optical-signal regeneration. This function is needed to overcome signal degradation due to physical layer impairments (PLI) which accumulate as light propagates through a network. Effects to be compensated are: loss, Amplified Spontaneous Emission (ASE) noise, chromatic and polarization- mode dispersion, filter cascading, non-linear effects such as four-wave mixing, self- and cross-phase modulation, Brillouin and Raman scattering. They are particularly severe in high-bitrate and long-haul Dense-WDM (DWDM) systems.

* This work was partially supported by the Spanish Ministry of Science and Innovation under contracts TEC2008-02552-E and TEC2009-07041 as well as by the Catalan Government under contract 2009 SGR1508
The use of amplifiers, usually erbium-doped fiber amplifiers (EDFA) in order to compensate the losses produced along the nodes and fibers, may introduce some of these PLIs, such as the ASE noise and the crosstalk. The crosstalk is produced by the interference of two or more lightpaths traversing the same switching node and its noise is copropagate along with the signal over the assigned lightpath [1].

On the other hand, the most commonly-adopted solution to regenerate the optical signal is the opto-electronic regenerator (also known as "transponder). This device converts signals from optical to electronic form, regenerates, and converts back to optical. Since the regenerator breaks up the optical continuity, the optical network becomes a translucent optical network. Regeneration is essential to re-amplify, re-shape and re-time the optical signal (i.e., 3R or simply regeneration). The double conversion from optical to electronic and back to optical (OEO conversion) is highly power-consuming. They are among the most expensive network elements of an OTN. In order to save on CAPEX, regeneration capability can be sparsely provided in the network, installing transponders where needed, possibly trying to minimize their number.

The incorporation of the physical layer impairments that degrade the optical signal in the Routing and Wavelength Assignment (RWA) for transparent and translucent optical networks is known as Impairment Aware RWA or IA-RWA. A survey of IA-RWA algorithms in optical networks can be found in [2]. Moreover, off-line planning of a translucent network requires deciding where allocate the regenerators in an optimal way, on the basis of a given forecast traffic demand. This is known as regenerator placement. Once the network is in operation (with dynamic traffic), the IA-RWA process of a new connection has to take into account the location of the available regeneration points, trying to answer questions such as: where is it better to use a regenerator for that specific connection. This is known as regenerator allocation.

In this paper we evaluate the effect of considering the crosstalk effects when selecting lightpaths and when allocating regenerators. We enhance the physical model proposed in [3] with the inclusion of node crosstalk effects. Our new physical model is a simple model based on the crosstalk model proposed in [4]. Moreover, we evaluate the impact of the crosstalk on IA-RWA algorithms with and without regenerator allocation (usage) optimization. Finally, we evaluate the capacity of different wavelength assignation techniques to coun- 
teract the crosstalk effects. One of these techniques, Minimum Crosstalk interference, MCI, is a new proposal which is an adaptation to translucent networks of the proposal, MC, in [5].

The rest of the paper is organized as follows. Section II presents the proposed translucent OTN physical model. Section III describes the different IA-RWA algorithms, including wavelength assignation techniques, that later will be evaluated in Section IV. Finally Section V concludes the paper.

\section{THE TRANSLUCENT OTN MODEL}

In optical-circuit-switched networks the basic managed entity is the lightpath, an end-to-end optical connection between a source and a destination node, requiring one wavelengthchannel per crossed network link.

A lightpath is subject to physical layer impairments (linear and non-linear) which degrade its quality as it transparently propagates through the network: the physical-layer model allows us to relate such degradation to the physical parameters of the network elements crossed along the path. Our model is based on the following elements: optical-switching nodes, regenerators, transponders, and DWDM line systems. In a translucent OTN the optical-switching nodes are the sites where transmitters, receives and regenerators are located. The core of the node is a non-blocking all-optical switching fabric, assumed to introduce attenuation and crosstalk (i.e. interference between WDM channels). The input/output ports of the node are connected to tunable transponders that provide the appropriate color (wavelength) and power level to the optical signal Each DWDM line system is bidirectional and composed of: i) a couple of counter-propagating fibers; ii) the set of optical Erbium Doped Fiber Amplifiers (EDFAs) necessary to completely recover loss due to fiber propagation. An opticalamplifier span is the optical-fiber segment connecting an EDFA to the next one or to the end-node of the link. We assume that each EDFA is placed at the beginning of its span. The first EDFA of a link in one direction is located at the output of the source node of the link (i.e., it is used as a booster); all the other EDFAs of a link are used as line amplifiers.

If a lightpath can not be established transparently due to the physical layer impairments, a regenerator is added in a transit node and dedicated to the connection: the lightpath is split by each regenerator into two contiguous transparent sub-paths. We consider that transit signals are not directly processed by regenerators and/or converters located at the network ports, but rather, a pool of regenerators/converters can be connected at dedicated ports of the switching fabric to flexibly allow regeneration and wavelength conversion

At the end of each sub-path (except the last one) a regenerator (OEO transponder) renews the signal. In this work we are considering 3R (re-amplification, re-shaping and re-timing) regenerators, which fully restore signal quality as if at the transmitter. Thus, from a signal-impairment point of view, the regeneration operation implies a complete loss of memory of the history of the signal along the path followed to reach the regenerator.
On the other hand, the key parameter to measure the signal quality is the Bit Error Rate (BER), which directly contributes to the quality of service perceived by the user of a circuitswitched network: a lightpath can be set up if the BER at the receiver is above a threshold. A BER threshold translates by well-known relations into a threshold value of the so-called Personick $Q$ factor [6], which in turn can be evaluated as a function of the transmission-system parameters and the physical layer impairments.

The computation of the $Q$ factor can be carried out by different methods, from analysis in the simplest cases, to physicallayer simulations in the most complicated ones. In particular, we have adopted in our model a semi-empirical method proposed in [3]. The computation of the $Q$ factor in [3] takes the following impairments into account: ASE, loss (linear); selfand cross-phase modulation (non-linear). In this work we propose to also introduce the crosstalk effects. The other effects (and in particular, polarization-mode and chromatic dispersions) are not considered.

Typically, the requirements for the minimum value of $Q$ of a signal at the receiver are about $17 \mathrm{~dB}$ without error correction, which corresponds to a BER of approximately $1 \times 10^{-12}$.

\section{A. Q Personick's model}

Let us consider a $h$-hop sub-path p, crossing $h$ links having lengths $L_{(1, p)}, \ldots, L_{(\mathrm{h}, p)}$ from the source to the end node. The $Q$ factor at the end of the sub-path is given by the equation:

$$
Q_{p}[\mathrm{~dB}]=a_{0}+a_{1} \operatorname{OSNR}_{p}+a_{2} N_{p}+a_{3}\left(P_{0} \cdot N_{p}\right)^{B}
$$

According to (1) the quality factor depends both on linear and non-linear effects. The $O S N R_{p}$ factor is the optical signal to noise ratio expressed in $\mathrm{dB}$ at the receiver. The terms $a_{2} N_{P}$ and $a_{3}\left(P_{0} N_{P}\right)^{\mathrm{B}}$ take into account the non-linear effects, considering all amplifiers along a path (booster at the beginning of the line and line amplifiers at intermediate sites on the lines). $N_{p}$ is the total number of EDFA-amplifier spans crossed by the sub-path, which is given by: $N_{p}=\sum_{j=1}^{h}\left\lceil L_{(j, p)} / s\right\rceil$, where $s$ is the maximum span length, and all the spans of a link $j$ have equal length $L_{(j, p)} /\left\lceil L_{(j, p)} / s\right\rceil$. It is assumed that each span contributes to the non-linear effects and $P_{0}[\mathrm{dBm}]$ is the power level at the signal launch (typically $3 \mathrm{dBm}$ ). The coefficients $a_{0}, a_{1}, a_{2}, a_{3}$, and $B$, on the other hand, depend on the type of line systems used and should be tuned by an onfield measurement campaign. Typical values were proposed in [3]: $a_{0} \cong 0,4, a_{1} \cong 1, a_{2} \cong-0.04, a_{3} \cong 0.02$ and $B \cong 0.2$.

$O S N R_{p}$ is the optical signal to noise ratio over a fixed optical bandwidth (dependent on the bitrate and the modulation format of the transmitters). The OSNR can be calculated considering the OSNR across each of its elementary components and then combining the partial results. We consider as elementary components all the spans and the nodes along the (sub-)path. If $i$ indicates the generic component, $O S N R_{i}$ contribution is computed as follows: 


$$
\operatorname{OSNR}_{i}[\mathrm{~dB}]=P_{0}[\mathrm{dBm}]-Q N-\left|T_{i}\right|[\mathrm{dB}]-F_{i}[\mathrm{~dB}]
$$

where $Q N$ is the quantum noise (typical value is $-58 \mathrm{~dB}$ ), $F_{i}$ $[\mathrm{dB}]$ is the noise figure of the optical amplifier belonging to the component and $T_{i}$ is the total attenuation of the element in $\mathrm{dB}$. When $i$ refers to a span, $F_{i}$ is the noise figure of a line or a pre-amplifier (assumed to have the same characteristics); when $i$ indicates a node, $F_{i}$ is the noise figure of the booster amplifier located at the output of the node. Similarly, $T_{i}$ is the attenuation of the fiber for a span, and of the switching fabric for a node. The OSNR at the end of the transparent (sub-)path is then expressed in linear units by $R_{\text {Total }}$, computed as follows:

$$
O S N R_{\text {end }}=10 \log _{10}\left(R_{\text {Total }}\right), \quad \text { with } \frac{1}{R_{\text {Total }}}=\sum_{i}\left(\frac{1}{R_{i}}\right)
$$

where $R_{i}$ is the OSNR (in linear units) of the element $i$ of the (sub-)path $\left(R_{i}=10^{\mathrm{OSNR}_{i} / 10}\right)$. In this framework, (1) allows to estimate the end-to-end impairment of an optical connection traveling across the translucent OTN.

\section{B. Including Crosstalk Effects}

In this section we improve the Q Personick's model presented above by including the node crosstalk effects. The proposed crosstalk model is simple enough and with low complexity to be dynamically run by the online IA-RWA algorithm.

According to [4] the in-band crosstalk generated in the optical switching nodes is considered as a major physical impairment that significantly impact the BER. The in-band crosstalk is produced by the interference of WDM channels occupying the same or adjacent wavelengths when traversing an optical switching node. It takes into account self crosstalk, cowavelength crosstalk and neighbor-port crosstalk. Each type of crosstalk is modeled [7] as a noise which cumulates linearly along the path, i.e. the ratio between interference crosstalk power and signal power cumulates linearly at each node. The co-wavelength crosstalk is produced by lightpaths traversing the switching node with the same wavelength as the candidate lightpath; it depends on the fabric switching port crosstalk ratio parameter, $X_{\text {port }}$, of the optical node. The self cross-talk and the neighbor-port crosstalk is produced by the lightpaths traversing the node with wavelengths adjacent to the wavelength of the candidate lightpath, it depends on the adjacent channel crosstalk ratio parameter, $X_{a d j}$, of the node.

For a concrete lightpath the degradation in the BER depends on the number and intensity of crosstalk components along the path. According to eq. (3) the end linear OSNR can be added; each component contributes $\frac{\mathbf{1}}{\boldsymbol{R}_{\boldsymbol{i}}}$ to the inverse of the end linear OSNR. In a concrete optical node each lightpath with a wavelength which is identical to the wavelength of the candidate lightpath contributes $\frac{\mathbf{1}}{\boldsymbol{R}_{p o r t}}$ to the inverse end linear OSNR; where $R_{\text {port }}$ is the switch port crosstalk ratio in linear units. Moreover each lightpath with a wavelength which is adjacent to the wavelength of the candidate lightpath contributes $\frac{\mathbf{1}}{\boldsymbol{R}_{a d j}}$ to the end linear OSNR; where $R_{a d j}$ is the adjacent crosstalk ratio in linear units. Then for an optical node $\mathrm{i}$ we can compute the linear OSNR produced by the crosstalk effects, $\mathrm{R}_{\mathrm{i} \_\mathrm{XT}}$, as:

$$
\frac{1}{R_{i-X T}}=\frac{N_{i}^{p o r t}}{R_{p o r t}}+\frac{N_{i}^{a d j}}{R_{a d j}}
$$

where $\boldsymbol{N}_{\text {port }}^{i}$ is the number of lightpaths in the node with the same wavelength as the candidate lightpath and $\boldsymbol{N}_{a d j}^{i}$ is the number of lightpaths in the node with a wavelength adjacent to the wavelength of the candidate lightpath. Then for a transparent lightpath with $\mathrm{M}$ nodes the total contribution of the crosstalk to the inverse of the end linear OSNR is:

$$
\frac{1}{R_{X T}}=\sum_{i=1}^{M} \frac{1}{R_{i-X T}}
$$

which is added to the total OSNR according to eq. (3).

\section{IA-RWA ALGORITHMS TAKING INTO ACCOUNT THE CROSSTALK EFFECTS}

In order to evaluate the network performance when the crosstalk effects are taken into account, we carry out a set of simulations with different IA-RWA algorithms.

First of all we compare the performance of the IA-RWA algorithms that try to optimize the use of regenerators against the IA-RWA algorithms without regenerator allocation optimization. The regenerator allocation consists on selection which of the already installed regenerators should be used in order to maximize the optical quality of the signal while minimizing the use of the regenerators. In this scenario, the IA-RWA algorithm selects the path and also the nodes of the path where the signal must be regenerated, diving the path in sub-paths between nodes with 3R regenerators. Moreover, the IA-RWA algorithm must use a wavelength selection technique in order to select the wavelength (or wavelengths) in the path (or in each sub-path).

In [8] the K-SP-REG algorithm is proposed. It selects among the $\mathrm{K}$ shortest path the shortest available, then it allocates only the needed regenerators along the selected path (only in the nodes where the quality of the optical signal falls below a threshold). Whereas the K-SP algorithm, without regenerator allocation optimization, allocates all the regenerators that it finds in the nodes along the selected path. Both algorithms utilize the First-Fit (FF) wavelength selection (K-SP-REG-FF and K-SP-FF in the performance evaluation).

On the other hand, we also compare the performance of the previous algorithms, K-SP-REG and K-SP when the wavelength selection is done randomly (K-SP-REG-Random and $\mathrm{K}-\mathrm{SP}-\mathrm{Random}$ ). In this case, the algorithm randomly picks one wavelength among the set of available wavelengths. This 
wavelength assignation technique is proposed in order to separate the assigned wavelengths and then to decrease the crosstalk effects.

Finally, we propose a new wavelength assignation algorithm, Minimum Crosstalk Interference (MCI) which is based on the MC algorithm proposed in [5] but in our case for translucent network. Once the path is selected and the corresponding regenerators allocated, this wavelength assignation algorithm runs as follows. For each wavelength, $j$, and for each sub-path, $s$, in the path, it computes the crosstalk intensity factor, CI (Eq.6).

$$
C I_{j}^{s}=\sum_{i=1}^{M_{s}}\left(R_{p o r t} \times N_{i}^{p o r t}+R_{a d j} \times N_{i}^{a d j}\right)
$$

where $j$ is the considered wavelength, $s$ the considered subpath, $\mathrm{R}_{\text {port }}$ is the switch port crosstalk ratio in linear units, $\boldsymbol{N}_{\text {port }}^{i}$ is the number of lightpaths in the node with the same wavelength $j, \mathrm{R}_{\text {adj }}$ is the adjacent crosstalk ratio in linear units and $\boldsymbol{N}_{a d j}^{i}$ is the number of lightpaths in the node with a wavelength adjacent to wavelength $j$.

The CI of the path will be the maximum CI among the subpaths of the path (Eq.7).

$$
C I_{j}=\max \left(C_{j}^{s}\right) \forall s
$$

Finally, it selects the wavelength minimizing the CI value (Eq.8).

$$
C I=\min \left(C_{j}\right) \forall j
$$

In the performance evaluation section we show also results of the K-SP-REG-MCI and K-SP-MCI algorithms.

\section{PERformance EVAluation}

\section{A. Simulation set up}

The network used in this study is the Pan European network with 28 nodes and 41 links. All links are equipped with sys- tems of $W=40$ wavelengths each, each WDM channel modulated at $10 \mathrm{Gbit} / \mathrm{s}$ (assuming an optical bandwidth per channel of $0.1 \mathrm{~nm}$ ). The number of systems installed in parallel on each link is calculated by the design procedure in a previous phase. We have in total 56 installed systems in the 41 links of the network, and 14 regenerators in 14 of the 28 nodes of the network.

The dynamic demand consists of an offered traffic ranged between 0.1 and 1 Erlang between each pair of nodes (i.e 1 Erlang corresponds to a total of 378 Erlang in the Network). The dynamic traffic is assumed to have an exponential distribution both in the inter-arrival and holding time. We simulated 37800 optical connection requests.

We set the following parameter of the simulation: $X_{\text {port }}$ and $X_{a d j}$ to $-30 \mathrm{~dB}$ and $-25 \mathrm{~dB}[5]$ respectively which means $\mathrm{R}_{\text {port }}=$ 0.001 and $\mathrm{R}_{\mathrm{adj}}=0.0032$ in linear units; and also we set $\mathrm{K}=2$ (for K-SP).

\section{B. Crosstalk effect}

In this first set of simulation we evaluate the effect of misconsidering the crosstalk effect in the IA-RWA algorithms. We assume that the K-SP and the K-SP-REG algorithms compute the quality of the signal factor, $Q$, according to the $Q$ Personick's model presented in Section II A. (without taking into account the crosstalk effect). Then, these algorithms compute the Q Personick's value of each sub-path and select the path and wavelength; and allocate the regenerators in the nodes utilizing this $Q$ value. However, we also assume that the real $Q$ values in the networks depend on the crosstalk produced by the interference of the different wavelengths; and is computed according to the model presented in Section II B. In this scenario, the computed $Q$ value differs from the real $Q$ value, and in some cases the lightpath assignation or/and regenerator allocation may fail. The source node can select a lightpath with a computed $Q$ value which is high enough, but when the lightpath is trying to be set up the connection can be blocked because the real $Q$ value is lower than the threshold (due to the crosstalk effects).

Figure 1 shows the blocking probability versus the traffic load when the K-SP-FF (a) and K-SP-REG-FF are unaware of
K-SP-FF

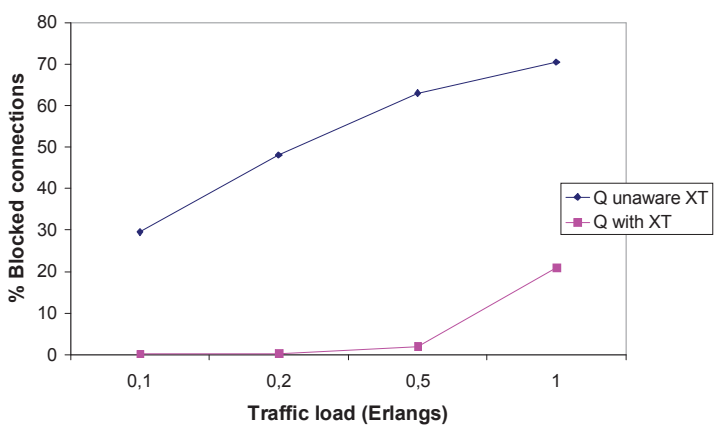

a) K-SP-FF algorithm
K-SP-REG-FF



b) K-SP-REG-FF algorithm

Figure 1. Comparison of blocking probability of the physical model unaware and considering of Cross-talk (XT). 


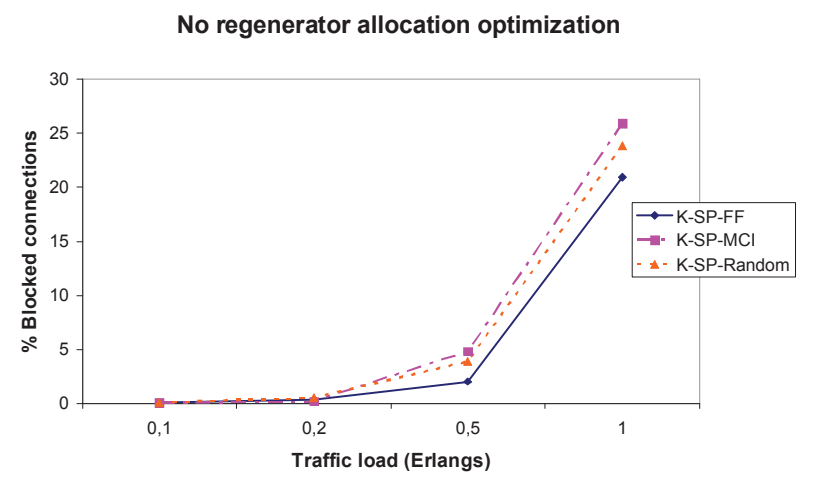

a) Blocking probability

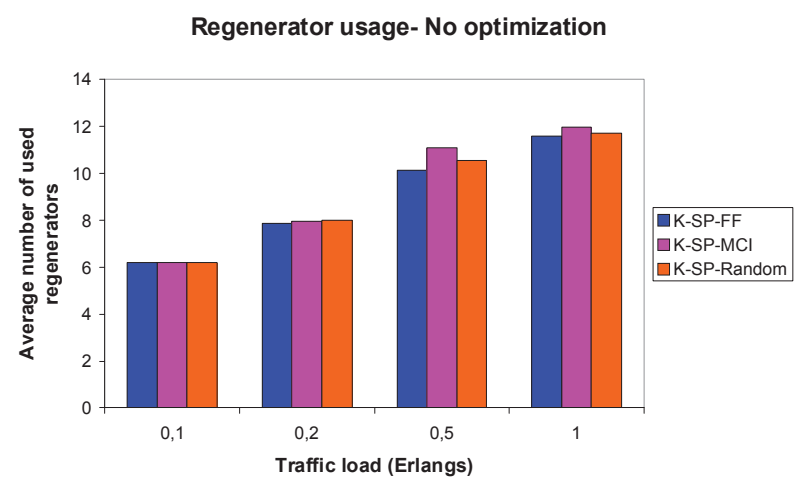

b) Average number of used regenerators

Figure 2. Blocking probability and average number of used regenerators for algorithms without regenerator allocation optimization.

the crosstalk effects and when they take into account these effects. We can observe first of all the best results of the algorithm that optimize the use of regenerators (K-SP-REG-FF) especially for low traffic load. We also observe that for both algorithms there is a higher blocking probability when the crosstalk effects are misconsidered; between a $30 \%$ and a $60 \%$ more of connections are blocked. In average for the different traffic loads 18000 more connections are blocked.

\section{Wavelength assignation comparison}

In the next set of simulation we compare the performance in terms of blocking probability of the different wavelength assignation techniques proposed in Section III: FF, Random and MCI. For these simulations the physical model considers the crosstalk effects according to the model presented in Section II B.

Figure 2 shows the comparison between the 3 wavelength assignation techniques, when the algorithm does not optimize the allocation of regenerators; in terms of a) blocking probability and b) regenerator usage. K-SP-FF presents the best performance in terms of blocking probability and also in average number of used regenerators. Moreover the random assignation does not improve the results obtained by the First Fit assignation.
Figure 3 shows the same results as Figure 2 but for the algorithms that optimize the use of the regenerators. We can see that the algorithms with regenerator optimization usage perform better in terms of blocking probability than without optimization (especially for low traffic load, between 0.1 and 0.5 Erlangs). If a connection does not need regeneration in an intermediate node of the path the regenerator is not used and then it is available for a different connection. Moreover the average number of used regenerators is lower with optimization and the energy consumption is reduced. The three wavelength assignation techniques, FF, random and MCI, perform very similar for low traffic load ( 0.1 to 0.5 Erlangs). For high traffic load (1 Erlang) the MCI assignation performs the worst in terms of blocking probability and average number of used regenerators; this is due because for this traffic load a high percentage of blocked connections are produced by the wavelength unavailability (there is not enough wavelengths) and the MCI assignation is not useful in this case.

For high traffic load (1 Erlang) Figure 4 shows the percentage of blocked connections produced by wavelength unavailability or by $Q$ unavailability (because the selected lightpath does not have enough quality) of FF, Random and MCI, for a) 14 installed regenerators and b) 28 installed regenerators. We observe the reduction of the whole percentage of blocked
Regenerator allocation optimization

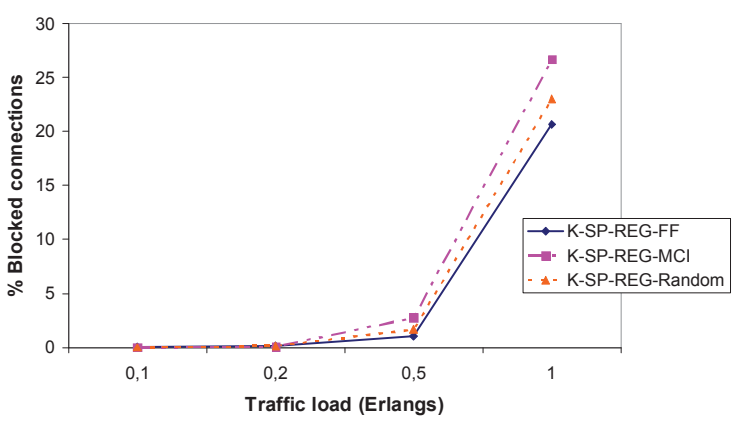

a) Blocking probability

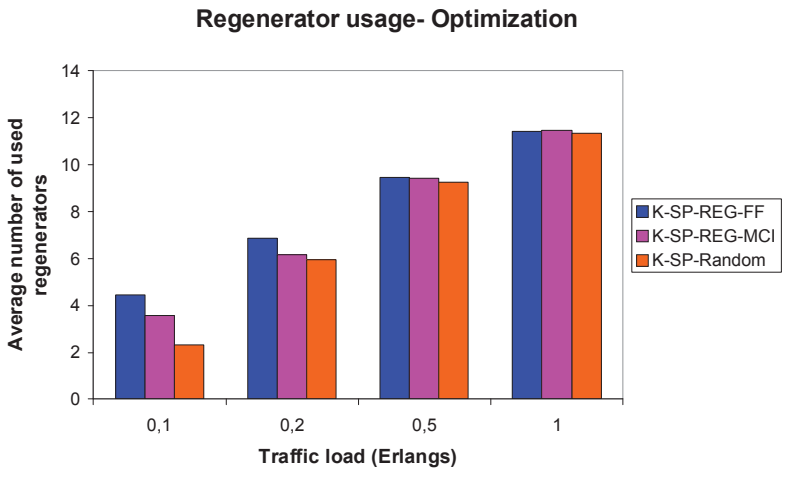

b) Average number of used regenerators

Figure 3. Blocking probability and average number of used regenerators for algorithms with regenerator allocation optimization. 
connections when we have more regenerators. This is because the number of connections that are blocked for $Q$ unavailability is reduced. However we can deduce that the main cause of blocked connections is the lack of wavelengths because when more connections have enough quality (increasing the number of regenerators) and would be able to be established there are more blocked connection for lacking of wavelengths (Figure 4 b)).

Finally, FF also performs the best for high traffic load. We can conclude that the FF assignation technique is good enough even when we consider the crosstalk effects in the physical mode.

\section{CONCLUSION}

In this paper we have shown the effect of misconsidering the crosstalk effect when assigning routes and wavelengths; and when allocating regenerators. More connections are blocked when the IA-RWA algorithm utilize a physical model that does not include the interference between lightpaths (crosstalk)

We have improved an initial physical mode in order to include the switching node crosstalk effects. Our proposed model is simple enough and it is based on the knowing of the switch port and adjacent wavelength crosstalk ratio.

One the IA-RWA utilize a physical model taking into account the crosstalk, we have studied different wavelength assignation techniques and their benefits when having crosstalk. One of the proposed techniques, Minimum Crosstalk Interference is a new, proposal that selects wavelengths producing the less interference with the already established lightpaths.

From the results we conclude that the IA-RWA algorithms with regenerator allocation optimization perform better for all the different wavelength assignation techniques. And also we observe that First Fit wavelength assignation performs very similar to the new proposed MCI; and then First Fit is a good and simple solution even when the crosstalk effects are considered.

\section{REFERENCES}

[1] B. Ramamurthy, D. Datta, H. Feng, J.P. Heritage, B. Mukherjee, "Impact of Transmission Impairments on the Teletraffic Performance of Wavelength-Routed Optical Networks", Journal of Lightwave Technology, Vol.17, N.10, October 1999.

[2] S. Azodolmolky, M. Klinkowski, E. Marin, D. Careglio, J. Sole Pareta, I. Tomkos., "A Survey on Physical Layer Impairments Aware Routing and Wavelength Assignment Algorithms in Optical Networks", Computer Networks, Vol. 53, issue 7, pp. 926-944, May 2009.

[3] M. Yannuzzi, M. Quagliotti, G. Maier, E. Marin-Tordera, X. MasipBruin, S. Sanchez-Lopez, J. Sole-Pareta, W. Erangoli, and G. Tamiri, "Performance of translucent optical networks under dynamic traffic and uncertain physical-layer information," in Proceedings of the 13th IFIP/IEEE Conference on Optical Network Design and Modelling (ONDM 2009), Braunschweig, Germany, February 2009.

[4] T. Deng, S. Subramaniam, J. Xu, "Crosstalk-Aware Assignment in Dynamic Wavelength-Routed Optical Networks", First International Conference on Broadband Networks (BROADNETS'04), San José, USA.

[5] Y. Zhai, Y. Pointurier, S. Subramaniam, M.Brandt-Pearce, "QoS aware algorithms for path-protected DWDM networks", Optical Fiber Communication conference, OSA/OFC 2007, Los Angeles, USA.

[6] S.D. Personick, "Receiver Design for Digital Fiber Optic Communication Systems, I", Bell Syst. Tech. J, Vol. 52, No. 6, July-August, 1973, pp. 843-874.

[7] N. Sambo, N. Andriolli, a. Giorgetti, F. Cugini, L. Valcarenghi, P. Castoldi, "Introducing Crosstalk-awarness inot GMPLS-controlled transparent optical networks", Optical Fiber Communication conference, OSA/OFC 2008, San Diego, USA.

[8] E. Marín Tordera, R. Martínez, R. Muñoz, R. Casellas, J. Solé Pareta, "Improving IA-RWA algorithms in translucent networks by regenerator allocation," in Proceedings of 11th International Conference on Transparent Optical Network, ICTON 2009 Açores, Portugal.

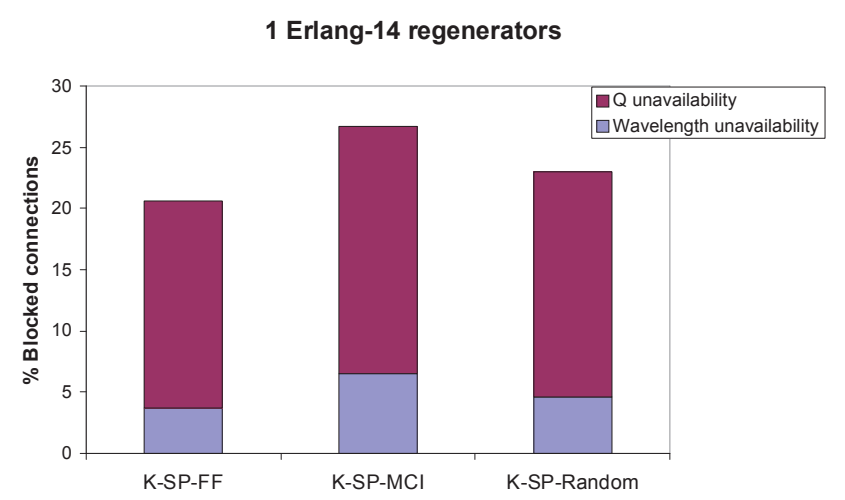

a) With 14 regenerators

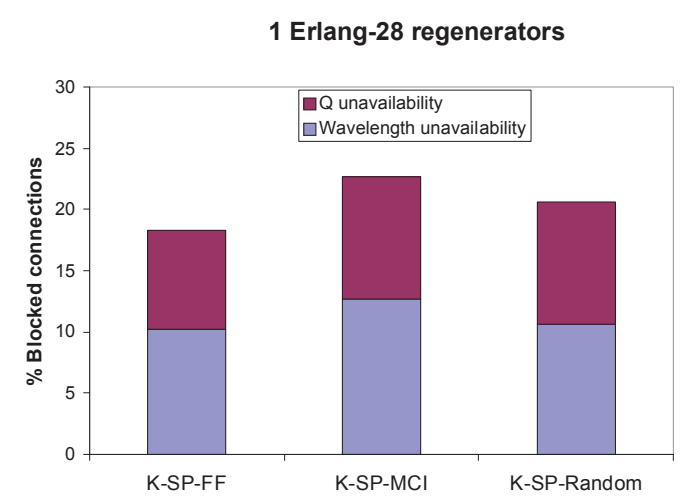

b)With 28 regenerators

Figure 4. Blocking probability caused by wavelength unavailability and physical $(Q)$ unavailability. 